\title{
Penser le Monstre par le biais du Discours Littéraire
}

Junia Barreto

Résumé

Dès l'origine, I'homme ne cessa de créer des monstres et de leur prêter des significations. Face aux aberrations de la forme ou de l'esprit, venues de l'imaginaire, produites par la nature ou par la société, il éprouve des sentiments fort ambigus. Au-delà des certitudes, l'écriture éternisa des monstres de toutes sortes, qui trouvent dans la littérature un champ fécond d'expression et de perpétuation.

Mots-clés: Monstre, différence, étrangeté, aberration, déviance.

Comment définir et où situer le monstre dans l'univers à partir des interprétations de l'homme sur le monde et l'origine des êtres? Est-ce qu'íl est le même ou l'autre de I'homme ou encore serait-il quelque chose entre les deux? Est-ce la créature monstrueuse, un mixte d'esprit et de matière? Est-ce qu'il se situe dans une zone intermédiaire de l'humanité de l'homme, entre l'imaginaire et la réalité? De nombreuses questions de ce genre se posent à tout instant quand on s'intéresse à la problématique du monstre et de la monstruosité.

Les définitions de monstre et de monstruosité peuvent varier selon les époques, selon les diverses perspectives: sociologique, philosophique, psychologique et encore selon les auteurs qui s'intéressent au sujet. La monstruosité peut également se révéler sous des formes distinctes, soit dans l'apparence des êtres, soit dans leurs caractères ou leurs actes. Elle peut se manifester chez les êtres humains, comme on peut la reconnaître chez les animaux, les entités de la nature et de la société ou encore chez les êtres purement fantastiques et les prodiges, produits par l'imagination humaine.

Nous réfléchissons aux monstres probablement depuis toujours, car ils font partie de la nature et de l'imaginaire humain, rongé par les peurs, les désirs et les sentiments les plus cachés et les plus étranges. Tout ce qui entoure le monstre et le monstrueux est lié à l'étrange, à l'inconnu et à l'énigmatique. Le monstre nous oblige à poser des questions pour lesquelles nous n'avons pas forcément de réponses. Et c'est justement par le moyen des récits, des légendes et des textes littéraires que les figures monstrueuses sont passées et se sont perpétuées au long des temps jusqu'à nos jours, laissant leurs empreintes, le questionnement sur leur existence et l'inquiétude dérangeante engendrée par leur difformité, qu'elle soit de caractère physique ou moral. Toute la problématique concernant le monstre renvoie l'homme à ses propres questionnements sur la nature des êtres, sur l'existence du bien et du mal, sur 
l'existence de Dieu lui-même et sur la validité et la propriété des normes et des conceptions sociales créées, telles que le beau et le laid, le vrai et le faux, le bon et le mauvais ou le correct et l'incorrect. Une chose est certaine: quel que soit le point de vue, le monstre reste toujours au-delà des normes et il bouleverse tous les paramètres convenus de la normalité, soit du côté de la forme, soit du côté du comportement.

La définition du monstre (ainsi que les paramètres qui définissent la monstruosité) et les rapports entre l'homme et le monstre n'ont pas été les mêmes au fil des siècles. Si nous prétendons étudier les monstres de manière plus scientifique, il faut les analyser à l'intérieur de la matrice inextricable de relations qui les génèrent: sociales, culturelles et littéro-historiques, puisqu'ils échappent à toute tentative de catégorisation facile. Le monstre se refuse à appartenir à l'ordre classificatoire des choses, résistant ainsi à tous les efforts qui l'incluent dans une structuration systématique quelconque. Il va questionner la pensée binaire, tout en y introduisant une crise. Le monstre est donc différence, polymorphisme, mélange, diversité, questionnement.

Il n'y a pas qu'une seule définition du monstre. Ce dont nous disposons ce sont des essais de définition, qui varient selon les théoriciens, les époques, les auteurs et l'utilisation du mot. Nous nous trouvons alors face à un concept extrêmement variable, nous permettant de passer de l'imaginaire au réel, de la mythologie à la science, du physique au moral et du sens propre au sens figuré du mot. Mais "le monstre raconte, comme en caricature, la genèse des différences ${ }^{1} \gg$. Généralement le monstre est défini par rapport à la norme. Cette norme est toujours un postulat du sens commun, ce qui fait que la définition dépend de la manière dont la norme est établie.

Le monstre n'appartient pas toujours à l'univers de la raison et maintes fois nous nous trouvons aussi face à l'imaginaire. Les différentes figures aperçues comme monstrueuses, qu'elles soient porteuses de difformité physique ou morale, évoquent quelque chose qui dépasse ou qui contredit la nature et les pouvoirs humains. L'attitude de I'homme face à la différence du monstre met en évidence l'ambiguïté de la relation de ce couple indissociable: le monstre effraye et séduit, dans la mesure où l'homme le craint et le vénère. Le monstre invite l'homme à un espace inconnu et interdit, où celui-ci ne résiste pas à la tentation d'entrer. Quand I'homme regarde le monstre, il est en train de se procurer une image (un lieu) stable de lui-même. Cet étranger peut transformer en incertitudes toutes les certitudes de I'homme, ouvrant une brèche dans le probable-ou l'improbable-et permettant ensuite tous les types de conduites et de déviations. Le monstre ne peut pas être étudié comme 'un complément inversé et symétrique de l'humanité de l'homme', comme il était vu à la lumière de Descartes. La naissance d'un corps monstrueux rend évident le germe de l'inhumanité (car anomalie de la forme) dans l'humanité de l'homme. L'angoisse instaurée par le monstre nous oblige à prendre conscience d'une certaine appartenance à l'univers monstrueux, que l'homme essaie toujours d'oublier. C'est comme si l'imprévisible et l'épouvantable, selon José Gil, la bête, nous laisse entendre Victor Hugo, restaient cachés dans l'homme, mais toujours prêts à se manifester. C'est comme s'il y avait une frontière tracée, on ne sait pas où, au-delà de laquelle se désintègrent les paramètres identifiés comme humains. Le monstre serait alors un phénomène interstitiel, un produit de la contamination entre l'ontologie humaine et ce qui n'est pas perçu comme naturel.

Pour qu'un monstre devienne mythe-nous parlons de difformités dans l'apparence - il ne faut pas simplement qu'il ait des formes anormales, car les anomalies sont nombreuses mais les anomalies élues sont assez rares. Selon Wolff- 
Quenot, le monstre doit avoir un certain profil pour devenir un mythe: "une apparence fascinante et néanmoins organisée; un rappel plus au moins discret du monde animal; un lien réel et supposé avec les éléments; l'incarnation aisée d'un problème psychologique ${ }^{2} \gg$. Être monstre implique normalement une naissance hors du commun, que ce soit la naissance mythique d'un monstre réel ou la genèse d'un monstre mythique, l'importance ne demeure pas dans la distinction entre réel ou imaginaire, mais entre le profane et le sacré. Si quelqu'un naît monstre ou s'il le devient, une intervention divine en est normalement la cause. Les monstrueux cyclopes, par exemple, ont des parents qui sont des dieux, mais leur appartenance au domaine du divin est conséquence des lois d'hérédité. Les monstres humains ou demi-humains sont aperçus comme les restes d'une humanité ébauchée et incomplète, traduisant les hésitations et les essais du créateur. Il est toujours possible que là où il y a mystère éclose le mythe.

Une autre manière de fabriquer des monstres qui deviennent des légendes est à travers la métamorphose. Dans la mythologie gréco-romaine, la métamorphose des dieux et des hommes (leur changement total de forme et de nature) est un procédé commun à de nombreuses légendes et maintes fois cette transmutation est génératrice de monstres. Dans les anciennes fables qu'Ovide a recueillies dans son ouvrage Les Métamorphoses, ou un peu plus tard dans l'ouvrage du même nom d'Apulée ${ }^{3}$, I'homme est métamorphosé en astre ou en âne, ce qui témoigne toujours de la puissance d'un dieu bienveillant ou courroucé. Dans l'Antiquité, la métamorphose fonctionne également comme une explication poétique, aussi bien que symbolique et religieuse du monde.

Si la nature produit des monstres, c'est bien I'homme qui crée les mythes. Le mythe n'est rien d'autre qu'une histoire hors du commun ayant une valeur sacrée et le monstre est un être exceptionnel, du fait de son aspect unique et informe, auquel nous ne trouvons pas d'explications précises. La mythologie, travaillant sur des signaux, s'est emparée de ces naissances monstrueuses, considérées comme des signes et des avertissements, et les a traduits en son langage. Le mythe devient une première réponse donnée à I'humanité sur le pourquoi du surgissement de monstres. Le monstre mythique peut être vu comme un discours sur une forme, puisque le monstre appartient au domaine des formes et le mythe au domaine du discours.

Dans l'Antiquité, on nommait monstres les créatures désignées par la nature comme de véritables énigmes vivantes et contradictoires. Au centre d'une société où tout semblait être apparemment normal et dans les lieux établis (spatialement et spirituellement), le monstre se présentait comme une figure dérangeante, qui subvertissait l'ordre et rendait évident le pouvoir de la nature, tout en créant des intersections en ces lieux. Parmi les différentes religions et philosophies d'alors, on remarque un lien commun qui les unit: le principe opposé à la conception de l'unité créatrice. Les éléments de ce dualisme sont en lutte constante, l'un contre l'autre: le bien était responsable de la création parfaite des êtres et le mal, responsable des déformations et de la création des monstres. Chaque civilisation et tradition crée sa propre classification du monstrueux ou de l'anormal tout en essayant de répondre aux exigences du moment, en les immolant ou les divinisant.

Du Moyen Âge au XVIIIe siècle, le monstre était perçu surtout comme un mélange du royaume animal et du royaume humain. On croyait à l'origine bestiale des monstres, à un mélange de deux espèces, de deux individus, de deux sexes, de formes, de mort et de vie. On expliquait la monstruosité par la transgression des limites naturelles, des classifications et des lois. Elle se trouvait là où le désordre de la 
loi naturelle venait toucher, ébranler et inquiéter le droit: civil, canonique ou religieux. La polémique sur le monstre s'était installée au XVIIIe siècle, avec le surgissement de thèses en exposant leurs différentes explications scientifiques des naissances monstrueuses.

C'est à la fin du XVIIIe et au début du XIXe siècles qu'apparaît la figure du monstre moral, mettant en évidence les problèmes de conduite monstrueuse et de la criminalité monstrueuse. Si I'on croit que la criminalité est un élément nécessaire à la monstruosité, il y a une transformation de point de vue, et la monstruosité devient un qualificatif éventuel de la criminalité. Le monstre moral va alors émerger dans le domaine de la pratique judiciaire. Le monstre est le problème: c'est lui qui va interroger et le système médical et le système judiciaire. Il est la figure essentielle, autour de laquelle s'inquiètent et se réorganisent les instances du pouvoir et les champs du savoir. Selon Foucault, tout au long du XIXe siècle, on s'est intéressé au problème de la monstruosité humaine (dans le sens des déviations morales), dans l'espoir de découvrir ce qui existerait derrière les anomalies et les déviations. On commence alors à identifier l'individu anormal au monstrueux. Au fond, il est vu comme un monstre quotidien, un monstre banalisé.

La présence de monstres et l'exercice de la monstruosité ont beaucoup marqué le XXe siècle et, actuellement, aux débuts du nouveau millénaire, nous les voyons défiler partout, sous les formes les plus diverses, soit dans la réalité quotidienne (surtout chez les individus moralement difformes), soit dans l'imaginaire culturel. Les monstres sont devenus des figures du quotidien. Malgré une véritable banalisation et croissance de la violence partout dans le monde, de l'exhibition illimitée de la cruauté humaine et du dévoilement des anomalies de la personnalité, au contraire, le champ traditionnel des anomalies physiques s'est rétréci. Chaque jour nous identifions moins d'hommes, dont les pathologies pourraient s'inscrire dans le domaine tératologique, comme perçues autrefois.

Des figures monstrueuses appartenant à la société, à celles créées par l'imaginaire social, les exemples de monstres à I'heure actuelle pullulent. Des types physiquement ou psychologiquement difformes aux créatures fantastiquement abominables, jusqu'à celles actuellement fabriquées dans les laboratoires, génétiquement modifiées. Et il ne faut pas oublier les cyborgues et les machines technologiquement monstrueuses. Des monstres moraux exerçant leur pouvoir et la violence, comme Hitler et Milosevic; des monstres physiques représentés par les victimes des médicaments modernes, comme la thalidomide, les hormones, les psychotropes, les anticoagulants oraux; des monstres-machines, comme les tout nouveaux missiles à tête chercheuse, poursuivant leur cible jusqu'à la destruction totale, ou la matérialisation de la science-fiction sous la forme d'êtres clonés, qui dénoncent la symbiose entre la science et l'homme; et tous les monstres créés par la littérature, le cinéma ou la télévision. Des nains de Freaks, à I'Elephant Man, Dr. Hanibal, Terminator, Freddy Kruger ou encore Alien ou La Mouche et l'infinité des personnages monstrueux, dans la forme ou dans le fond, créés par le domaine littéraire ${ }^{4}$, réels ou imaginaires. Les monstres, aux côtés du sexe, représentent de nos jours l'appel le plus fort de la culture de masse. La prolifération de monstres de tous genres dans les plus divers domaines témoigne de l'attraction sans bornes qu'ils exercent et, en même temps, dévoile le doute de l'homme contemporain quant à sa propre humanité. Le monstre ne se situe pas en dehors du domaine de I'humain, mais il se trouve dans ses limites, aux frontières de son humanité, dans un lieu nébuleux entre réalité et imaginaire qu'on ne peut pas nommer et dont les places ne sont pas les 
mêmes pour tous, car chaque monstre en est un et chaque monstruosité possède ses propres particularités.

Le monstre n'appartient donc à aucun âge spécifique, aucun moment historique, aucun peuple, aucun courant philosophique particulier, aucun moyen d'expression artistique unique. Le monstre est partout, soit dans le monde réel soit dans l'imaginaire humain, à tous les moments, dans tous les domaines, présent dans toutes les formes de manifestation de la pensée, de la connaissance et de l'art. Sa multiplicité de formes, de caractéristiques et même de personnalité se reflète dans toutes les approches et dans toutes les questions qui émanent de la réflexion sur le sujet. Peuplant les légendes mythiques, passant par les textes sacrés, les études d'histoire naturelle, arrivant aux récits poétiques ${ }^{5}$ ou de science-fiction, le monstre ne cesse d'imposer sa nature insaisissable.

\footnotetext{
Notas

$\left.\right|_{1}$

M. Foucault, Les mots et les choses, Paris, Gallimard, 1966, p. 170.

${ }^{2}$ M-J. Wolff-Quenot, Des monstres aux mythes, Paris, Guy Trénadiel, 1996, p. 154.

${ }^{3}$ Plus récemment, on retrouve le procédé de la métamorphose chez Kafka, se servant de la reprise ironique de ce modèle mythique et transformant I'homme en cafard.

${ }^{4}$ Comme, par exemple, Gregor Sansa, chez Sartre; André Mersault, chez Camus; I'immoraliste d'André Gide et aussi le loup-garou de José Cândido de Carvalho et les monstres de Caio Fernando Abreu, Sérgio Santana, entre autres.

${ }^{5}$ Je pense surtout à l'œuvre de Victor Hugo, qui a inspiré ma recherche de doctorat, Les figures du monstre dans l'œuvre théâtrale et romanesque de Victor Hugo
} 
Abstract

Since the beginning, men create monsters and signify them. Facing those aberrations of form and spirit, produced by nature or society, men experience the most dubious feelings. Beyond certainty, writing eternalized all kinds of monsters, expressed and immortalized by literature.

Key words: Monster, difference, aberrations, strangeness, deviance.

\section{Referências}

CÉARD, Jean. La Nature et les prodiges: l'insolite au XVle siècle. 2.éd. Genève: Librairie Droz, 1996.

DURAND, Gilbert. As Estruturas antropológicas do imaginário: introdução à arquetipologia geral. Trad. de Hélder Godinho. São Paulo: Martins Fontes, 2002.

GIL, José. Monstros. Lisboa: Quetzal Editores, 1994.

FOUCAULT, Michel. Os anormais: curso no Collège de France (1974-1975). Trad. Eduardo Brandão, ed. sob a dir. de François Ewald e Alessandro Fontana. São Paulo: Martins Fontes, 2001.

FOUCAULT, Michel. Les Mots et les choses: une archéologie des sciences humaines. Paris: Éditions Gallimard, "Bibliothèque des Sciences Humaines», 1966. 400p.

GAGNEBIN, Murielle. Fascination de la laideur: l'en-deçà psychanalitique du laid, nouv. éd. augmentée d'une post-face. Seyssel: Éditions Champ Vallon "L'Or d'Atalane», 1994.

KAPPLER, Claude. Monstros, demônios e encantamentos no final da Idade Média. Trad. Ivone Castilho Benedetti. São Paulo: Martins Fontes, 1994.

LASCAULT, Gilbert. Le Monstre dans l'art occidental: un problème éthique. Paris: Éditions Klincksieck, 1973.

MARTIN, Ernest. Histoire des monstres: depuis l'antiquité jusqu'à nos jours. Grenoble: Éditions Jérôme Millon, "Mémoires du corps", 2002.

Monstre (Le) l: présence du monstre-mythe et réalité, Cahiers de recherche sur l'imaginaire, sous la direction de Jean Burgos. Paris: Lettes Modernes, 1975.

TORT, Patrick. L'ordre et les monstres: le débat sur l'origine des déviations anatomiques au XVIIle siècle. Paris: Syllepse, "Matériologiques", 1998.

WOLFF-QUENOT, Marie-Josephe. Des monstres aux mythes, préface d'Albert Jacquard. Paris: Guy Trédaniel Éditeur, 1996. 